\title{
Variation in biotic and abiotic factors associated with white spot syndrome virus (WSSV) outbreak in shrimp culture ponds
}

\author{
A. SWATHI, M. S. SHEKHAR, K. KARTHIC, K. VINAYA KUMAR, M. MURALIDHAR, \\ CHRIS HAUTON* AND K. K. VIJAYAN \\ Genetics and Biotechnology Unit, ICAR-Central Institute of Brackishwater Aquaculture, 75, Santhome High Road \\ R. A. Puram, Chennai, Tamil Nadu, India \\ ${ }^{*}$ Ocean and Earth Science, University of Southampton, National Oceanography Centre Southampton, SO14 3ZH \\ United Kingdom \\ e-mail:msshekhar@ciba.res.in
}

\begin{abstract}
Shrimp production in India has gradually increased since 2009 with the introduction of Penaeus vannamei. Most coastal states in India have been important contributors to $P$. vannamei production. However, among the many challenges faced in sustainable shrimp farming, prevention of viral diseases and pond water quality management remain major concerns. In this study, 27 shrimp ponds located in Kalpakkam and Elavur regions of Tamil Nadu were monitored to characterise the pond water parameters including $\mathrm{pH}$, salinity, temperature, alkalinity, ammonia, nitrite, hardness, dissolved oxygen (DO) and Vibrio spp. load. Sudden outbreaks of white spot syndrome virus (WSSV) were observed in some ponds which were found to be associated with variations in abiotic parameters. Parameters such as $\mathrm{pH}, \mathrm{DO}$ and nitrite were observed to be within the permissible range, while temperature, salinity, Vibrio spp. load and ammonia were relatively higher in certain ponds. The influence of these abiotic factors triggering WSSV outbreak were investigated in this study.
\end{abstract}

Keywords: Aquaculture, Penaeus vannamei, Vibrio spp., Water parameters, White spot syndrome virus

Shrimp farming in India is an important livelihood and economic activity. India alone exports $373866 \mathrm{t}$ of shrimp annually generating estimated revenue of 3096.68 million USD (MPEDA. 2016). Among the penaeid shrimps, Penaeus vannamei is a dominant species for aquaculture in India. This happened after a major policy decision was taken by the Govt. of India facilitating import of specific pathogen free (SPF) P. vannamei for domestic culture in India. Due to the availability of SPF seeds, scope for stocking at higher density, greater tolerance to salinity and rapid weight gain, many farms in India shifted to $P$. vannamei farming (Kumaran et al., 2017). Tamil Nadu (TN), the second largest shrimp producing state in India has approximately 7615 ha of $P$. vannamei cultivation producing $44453 \mathrm{t}$, with around 14 million people dependent on this activity and $P$. vannamei farming in TN has grown over $14.88 \%$ than the preceding year (MPEDA, 2016).

Among the many factors considered influencing shrimp growth, water quality is one of the most important criteria in addition to feeding, pollutants, diseases, soil quality, nutrients and use of extensive chemicals (Kautsky et al., 2000). Pond physicochemical characteristics affect shrimp growth, health and abundance (Penmetsa et al., 2013). In intensive and semi-intensive ponds, excessive water contaminants are observed mainly due to unconsumed feed and waste produced from cultured shrimps. Moreover, the presence of suspended solids, total ammonia, reactive orthophosphate, high stocking density, lack of proper aeration and excessive feeding also adversely affect the quality of pond water (Hopkins et al., 1993). Variations in water quality parameters lead to stress in the cultured shrimp stock (Bagarinao and Flores, 1995) which can predispose shrimp to opportunistic infection or exacerbate other outbreaks (Ananda Raja et al., 2012a).

It has been estimated that $60 \%$ of shrimp diseases are caused by viral pathogens and $20 \%$ by bacterial infestations (Flegel, 2012). The increasing level of stress directly attributes to diminished immune response and susceptibility to bacterial and viral infections (Le Moullac and Haffner, 2000; Tendencia et al., 2010; Ananda Raja et al., 2017b). Among many viruses affecting shrimp, white spot syndrome virus (WSSV) has been known to be highly lethal, causing mortality in a very short period of time (Ananda Raja et al., 2017a). The spread of WSSV infection occurs through contaminated water, seabirds, arthropods and other vectors. Shrimps are reported to be more susceptible to WSSV infection under high-stress conditions caused by poor pond management practises, water quality, food and other factors (Sanchez-Martinez 
et al., 2007). Significant economic loss also occurs as a result of outbreaks of Vibrio spp., which can become pathogenic under poor pond management practices (Gopal et al., 2005; Ananda Raja et al., 2017a). Therefore, it is important to identify these microbial and viral infections at an earlier stage to maintain a healthy stock. Hence in the present study, water quality parameters from 27 semi-intensive shrimp ponds were analysed to comprehend the interrelationship between pond management practices, water quality and shrimp health.

The data were gathered from 27 shrimp ponds in 13 farms at two different locations. The study areas comprised 20 ponds located in Kalpakkam region $\left(12.5238^{\circ} \mathrm{N} ; 80.1568^{\circ} \mathrm{E}\right)$ and 7 ponds located in Elavur region $\left(13.4628^{\circ} \mathrm{N}\right.$; $\left.80.1191^{\circ} \mathrm{E}\right)$ in Tamil Nadu. Sampling was done periodically for one winter crop in Kalpakkam (October - December 2016) and one summer crop in Elavur (March - June 2017). Pond management practices, shrimp health and pond water characteristics were regularly monitored and recorded.

The shrimp farms in Kalpakkam area ranged from 0.3 - 1.0 ha, while in Elavur region, pond sizes were about 0.2 - 0.4 ha. All the selected ponds cultured P. vannamei shrimps. The stocking density of each pond varied with respect to pond size (Table 4). PCR tested WSSV negative post-larvae (PL) were stocked in all ponds and fed with commercial pellet feed until harvest.

In Kalpakkam, shrimp farming was practised with regular water exchange while in Elavur, shrimps were acclimatised for zero water exchange. Water samples were collected in sterile plastic containers from below the pond surface during the culture period. Temperature, salinity (Refractometer RHS 10ATC), dissolved oxygen (DO) and $\mathrm{pH}$ (EcoTestr $\mathrm{pH} 1)$ were measured at the pond site. Shrimp samples were collected in sterile polyethylene bags and transported to the laboratory at $4^{\circ} \mathrm{C}$ for further analyses.

Physico-chemical properties of water viz., temperature, salinity, $\mathrm{pH}$, DO, alkalinity, hardness, ammonia, nitrite, Vibrio load as well as WSSV infection were monitored for the crop until harvest in shrimp farming ponds of Elavur and Kalpakkam. Shrimp samples were preserved in $-80^{\circ} \mathrm{C}$ freezer for further analysis. Water alkalinity was estimated by titration with strong acid until specific $\mathrm{pH}$ values were attained (MColortest ${ }^{\mathrm{TM}}$ Alkalinity test kit, Merck) and a colourimetric test was used to determine the nitrite level (MColortest ${ }^{\mathrm{TM}}$ Nitrite test kit, Merck). Detection of ammonium ions and dissolved ammonia was calculated based on colourimetric test and sliding comparator (Ammonium test kit, Merck).
Colourimetric test strips were used to measure the total hardness in pond water samples (Total hardness test kit, Merck). DO concentrations were estimated based on titration pipette and colour test using standard kit (MColortest oxygen test, Merck). Vibrio spp. load was estimated on thiosuphate citrate bile salt sucrose (TCBS) agar (HiMedia, Mumbai) plates (Biswas et al., 2012). The green and yellow colonies developed were counted manually. The gill tissues of preserved shrimp samples were dissected using sterile forceps and scissors and processed for DNA extraction (QIAamp DNA Mini Kit, QIAGEN). Detection of WSSV was done by PCR using WSSV specific primers (Durand et al., 1996).

The temperature was found to be in the range of 30 to $32^{\circ} \mathrm{C}$ for summer crop and 28 to $31^{\circ} \mathrm{C}$ for winter crop and significant variations were not observed between the two crops (Table 1, 2, 3 and 4). Kalpakkam ponds had salinity between 3 to $25 \%$ whereas Elavur ponds had salinity ranging between 10 to $24 \%$. The estimated $\mathrm{pH}$ value ranged from 7.1 to 8.9 in Kalpakkam shrimp ponds and 7.1 to 8.3 in Elavur ponds. In this study, $\mathrm{pH}$ was not observed to vary substantially. From the analysis of the semi-intensive shrimp ponds, DO concentration was in the range of 3 to $12 \mathrm{mg} \mathrm{l}^{-1}$. Data from our shrimp ponds in Kalpakkam recorded alkalinity in the range of 100 to $267 \mathrm{mg} \mathrm{l}^{-1}$ whereas shrimp ponds in Elavur region was in the range of 160 to $350 \mathrm{mg} \mathrm{l}^{-1}$. Hardness was estimated to be greater than $450 \mathrm{mg} \mathrm{l}^{-1} \mathrm{CaCO}_{3}$ equivalents from all these pond water samples. The absolute hardness value could not be determined in this study as the pond hardness was above the maximum range of $450 \mathrm{mg} \mathrm{l}^{-1}$ supported by the kit used (Total hardness test, Merck). Ammonia was found to be in the range of $0.01-1.0 \mathrm{mg} \mathrm{l}^{-1}$ and the ponds that tested positive for WSD were observed to have higher concentrations of ammonia ( 0.5 to $\left.1 \mathrm{mg} \mathrm{l}^{-1}\right)$. Eleven out of twenty seven ponds had high ammonia $\left(1 \mathrm{mg} \mathrm{l}^{-1}\right)$ levels. The concentration of nitrite in Kalpakkam shrimp ponds were 0.05 to $1.00 \mathrm{mg} \mathrm{l}^{-1}$ whereas in Elavur shrimp ponds the values were 0.01 to $1.00 \mathrm{mg} \mathrm{l}^{-1}$. Vibrio spp. load from these 27 shrimp ponds ranged between $10^{3}$ to $10^{6} \mathrm{cfu} \mathrm{ml}^{-1}$.

This study from 27 semi-intensive $P$. vannamei culture ponds summarises the interrelation of pond water quality, shrimp health and diseases that are observed in two locations in TN. The most common concern in maintaining water quality involve temperature, $\mathrm{pH}$, salinity, ammonia, nitrite, DO and hardness. Periodic monitoring of pond water parameters is important during the growing season prior to harvest (Cohen et al., 2005). In a recent study from Pokkali shrimp farms in the state of Kerala in India, which follow a system of cultivating paddy and shrimp 
Table 1.Water quality parameters recorded in shrimp ponds in Kalpakkam during first sampling

\begin{tabular}{|c|c|c|c|c|c|c|c|c|c|}
\hline Farm / Pond & $\begin{array}{l}\text { Pond size } \\
\text { (ha) }\end{array}$ & $\begin{array}{l}\text { Days of culture } \\
\text { (DOC) }\end{array}$ & $\mathrm{pH}$ & $\begin{array}{l}\text { Salinity } \\
(\%)\end{array}$ & $\begin{array}{l}\text { Alkalinity } \\
\left(\mathrm{mg} \mathrm{l}^{-1}\right)\end{array}$ & $\begin{array}{l}\text { Temperature } \\
\left({ }^{\circ} \mathrm{C}\right)\end{array}$ & $\begin{array}{l}\text { Total Ammonia } \\
\left(\mathrm{mg} \mathrm{l}^{-1}\right)\end{array}$ & $\begin{array}{l}\text { Nitrite } \\
\left(\mathrm{mg} \mathrm{l}^{-1}\right)\end{array}$ & $\begin{array}{l}\text { Vibrio load } \\
(\text { CFU ml-1) }\end{array}$ \\
\hline Farm A (Pond1) & 0.61 & 74 & 7.3 & 17 & 267.12 & 28 & 0.6 & 0.10 & $2.6 \times 10^{5}$ \\
\hline Farm A (Pond2) & 0.28 & 46 & 7.5 & 09 & 191.52 & 28 & 1.0 & 0.12 & $4.8 \times 10^{5}$ \\
\hline Farm B (Pond1) & 0.46 & 50 & 7.5 & 18 & 126.00 & 29 & 1.0 & 0.20 & $3.3 \times 10^{6}$ \\
\hline Farm B (Pond2) & 0.49 & 56 & 7.4 & 25 & 166.32 & 29 & 0.6 & 0.21 & $4.8 \times 10^{6}$ \\
\hline Farm B (Pond3) & 0.61 & 44 & 7.7 & 19 & 131.04 & 29 & 1.0 & 0.23 & $1.1 \times 10^{6}$ \\
\hline Farm C (Pond1) & 0.61 & 54 & 8.3 & 08 & 110.88 & 29 & 1.0 & 0.05 & $>6.5 \times 10^{6}$ \\
\hline Farm C (Pond2) & 1.01 & 61 & 8.1 & 11 & 141.12 & 29 & 1.0 & 0.05 & $>6.5 \times 10^{6}$ \\
\hline Farm C (Pond3) & 1.01 & 61 & 8.6 & 09 & 110.88 & 29 & 1.0 & 1.00 & $4.06 \times 10^{6}$ \\
\hline Farm D (Pond1) & 0.28 & 41 & 8.3 & 06 & 166.32 & 29 & 0.6 & 0.13 & $2.1 \times 10^{6}$ \\
\hline Farm D (Pond2) & 0.28 & 41 & 8.3 & 06 & 161.28 & 29 & 1.0 & 0.16 & $6.2 \times 10^{5}$ \\
\hline
\end{tabular}

*Hardness from farm A, B, C, D were above $450 \mathrm{mg} \mathrm{l}^{-1} \mathrm{CaCo}_{3}$

Table 2. Water quality parameters recorded in shrimp ponds in Kalpakkam during second sampling

\begin{tabular}{|c|c|c|c|c|c|c|c|c|c|c|c|}
\hline Farm/Pond & $\begin{array}{l}\text { Pond } \\
\text { size (ha) }\end{array}$ & $\begin{array}{l}\text { Days of } \\
\text { culture } \\
\text { (DOC) }\end{array}$ & $\mathrm{pH}$ & $\begin{array}{l}\text { Salinity } \\
(\%)\end{array}$ & $\begin{array}{l}\text { Alkalinity } \\
\left(\mathrm{mg} \mathrm{l}^{-1}\right)\end{array}$ & $\begin{array}{l}\text { Temperature } \\
\left({ }^{\circ} \mathrm{C}\right)\end{array}$ & $\begin{array}{l}\text { Total ammonia } \\
\left(\mathrm{mg} \mathrm{l}^{-1}\right)\end{array}$ & $\begin{array}{l}\text { Nitrite } \\
\left(\mathrm{mg} \mathrm{l}^{-1}\right)\end{array}$ & $\begin{array}{l}\text { Vibrio load } \\
\left(\mathrm{CFU} \mathrm{\textrm {ml } ^ { - 1 }}\right)\end{array}$ & $\begin{array}{l}\text { PCR test } \\
\text { for WSSV }\end{array}$ & Remarks \\
\hline $\begin{array}{l}\text { Farm A } \\
\text { (Pond1) }\end{array}$ & 0.61 & - & - & - & - & - & - & - & - & Positive & $\begin{array}{l}\text { Harvested } \\
\text { due to WSSV } \\
\text { outbreak in } \\
\text { nearby pond }\end{array}$ \\
\hline $\begin{array}{l}\text { Farm A } \\
\text { (Pond2) }\end{array}$ & 0.28 & - & - & - & - & - & - & - & - & Positive & $\begin{array}{l}\text { Harvested } \\
\text { due to WSSV } \\
\text { outbreak in } \\
\text { nearby pond }\end{array}$ \\
\hline $\begin{array}{l}\text { Farm B } \\
\text { (Pond1) }\end{array}$ & 0.46 & 62 & 7.2 & 20 & 226.80 & 30 & 1.0 & 0.05 & $>6.5 \times 10^{6}$ & Negative & \\
\hline $\begin{array}{l}\text { Farm B } \\
\text { (Pond2) }\end{array}$ & 0.49 & 68 & 7.1 & 20 & 105.84 & 30 & 0.6 & 0.05 & $>6.5 \times 10^{6}$ & - & \\
\hline $\begin{array}{l}\text { Farm B } \\
\text { (Pond3) }\end{array}$ & 0.61 & 56 & 7.1 & 23 & 105.84 & 30 & 1.0 & 0.1 & $>6.5 \times 10^{6}$ & - & \\
\hline $\begin{array}{l}\text { Farm C } \\
\text { (Pond 1) }\end{array}$ & 0.61 & 66 & 7.2 & 8.0 & 176.40 & 30 & 0.6 & 0.05 & $>6.5 \times 10^{6}$ & Negative & \\
\hline $\begin{array}{l}\text { Farm C } \\
\text { (Pond 2) }\end{array}$ & 1.01 & 73 & 7.4 & 9.0 & 151.20 & 30 & 1.0 & 0.15 & $>6.5 \times 10^{6}$ & Negative & \\
\hline $\begin{array}{l}\text { Farm C } \\
\text { (Pond 3) }\end{array}$ & 1.01 & 73 & 7.3 & 9.0 & 196.56 & 30 & 1.0 & 1.0 & $>6.5 \times 10^{6}$ & Negative & \\
\hline $\begin{array}{l}\text { Farm D } \\
\text { (Pond 1) }\end{array}$ & 0.28 & 53 & 7.7 & 7.5 & 226.80 & 30 & 1.0 & 0.05 & $>6.5 \times 10^{6}$ & Negative & \\
\hline $\begin{array}{l}\text { Farm D } \\
\text { (Pond 2) }\end{array}$ & 0.28 & 53 & 7.9 & 6.0 & 221.76 & 30 & 0.6 & 0.05 & $6.2 \times 10^{5}$ & Negative & \\
\hline $\begin{array}{l}\text { Farm D } \\
\text { (Pond 3) }\end{array}$ & 0.61 & 45 & 8.0 & 7.0 & 201.60 & 30 & 0.2 & 0.05 & $1.5 \times 10^{6}$ & Negative & \\
\hline $\begin{array}{l}\text { Farm D } \\
\text { (Pond 4) }\end{array}$ & 0.28 & 45 & 8.0 & 5.0 & 236.88 & 30 & 0.6 & 0.25 & $1.3 \times 10^{6}$ & - & \\
\hline $\begin{array}{l}\text { Farm D } \\
\text { (Pond 5) }\end{array}$ & 0.46 & 45 & 7.6 & 5.0 & 151.20 & 30 & 0.4 & 0.15 & $2.02 \times 10^{6}$ & - & \\
\hline $\begin{array}{l}\text { Farm E } \\
\text { (Pond 1) }\end{array}$ & 0.49 & 7 & 8.8 & 3.0 & 161.28 & 30 & 0.05 & 0.13 & $5.2 \times 10^{6}$ & Negative & \\
\hline $\begin{array}{l}\text { Farm E } \\
\text { (Pond 2) }\end{array}$ & 0.61 & 7 & 8.9 & 3.0 & 100.80 & 30 & 0.05 & 0.16 & $1.0 \times 10^{6}$ & - & \\
\hline
\end{tabular}

"Hardness from farm A, B, C, D were above $450 \mathrm{mg} \mathrm{l}^{-1} \mathrm{CaCo}_{3}$ 
Table 3. Water quality parameters recorded in shrimp ponds in Kalpakkam during third sampling

\begin{tabular}{|c|c|c|c|c|c|c|c|c|c|c|c|}
\hline $\begin{array}{l}\text { Farm/ } \\
\text { Pond }\end{array}$ & $\begin{array}{l}\text { Pond size } \\
\text { (ha) }\end{array}$ & $\begin{array}{l}\text { Days of } \\
\text { culture (DOC) }\end{array}$ & $\mathrm{pH}$ & $\begin{array}{l}\text { Salinity } \\
(\%)\end{array}$ & $\begin{array}{l}\text { Alkalinity } \\
\left(\mathrm{mg} \mathrm{l}^{-1}\right)\end{array}$ & $\begin{array}{l}\text { Temperature } \\
\left({ }^{\circ} \mathrm{C}\right)\end{array}$ & $\begin{array}{l}\text { Total ammonia } \\
\left(\mathrm{mg} \mathrm{l}^{-1}\right)\end{array}$ & $\begin{array}{l}\text { Nitrite } \\
\left(\mathrm{mg} \mathrm{l}^{-1}\right)\end{array}$ & $\begin{array}{l}\text { Vibrio load } \\
(\text { CFU ml-1) }\end{array}$ & $\begin{array}{l}\text { PCR test } \\
\text { for WSSV }\end{array}$ & Remarks \\
\hline $\begin{array}{l}\text { Farm D } \\
\text { (Pond4) }\end{array}$ & 0.28 & 60 & 8.1 & 4 & 252.00 & 31 & 0.50 & 0.24 & $>6.5 \times 10^{6}$ & Positive & \\
\hline $\begin{array}{l}\text { Farm D } \\
\text { (Pond5) }\end{array}$ & 0.46 & 60 & 7.8 & 5 & 231.84 & 31 & 0.20 & 0.14 & $>6.5 \times 10^{6}$ & Negative & \\
\hline $\begin{array}{l}\text { Farm E } \\
\text { (Pond 1) }\end{array}$ & 0.49 & 23 & 8.7 & 5 & 100.80 & 31 & 0.14 & 0.14 & $>6.5 \times 10^{6}$ & Positive & $\begin{array}{l}\text { High } \\
\text { mortality rate }\end{array}$ \\
\hline $\begin{array}{l}\text { Farm E } \\
\text { (Pond 2) }\end{array}$ & 0.61 & 23 & 8.9 & 5 & 105.84 & 31 & 0.20 & 0.20 & $8.0 \times 10^{3}$ & Negative & $\begin{array}{l}\text { High } \\
\text { mortality rate }\end{array}$ \\
\hline $\begin{array}{l}\text { Farm F } \\
\text { (Pond 1) }\end{array}$ & 0.61 & 25 & 8.9 & 5 & 126.00 & 31 & 0.20 & 0.20 & $>6.5 \times 10^{6}$ & Negative & \\
\hline $\begin{array}{l}\text { Farm F } \\
\text { (Pond 2) }\end{array}$ & 0.61 & 25 & 8.7 & 5 & 141.12 & 31 & 0.18 & 0.18 & $>6.5 \times 10^{6}$ & - & \\
\hline $\begin{array}{l}\text { Farm F } \\
\text { (Pond 3) }\end{array}$ & 1.01 & 25 & 7.8 & 6 & 226.80 & 31 & 0.24 & 0.24 & $1.1 \times 10^{5}$ & - & \\
\hline $\begin{array}{l}\text { Farm F } \\
\text { (Pond 4) }\end{array}$ & 1.01 & 25 & 8.7 & 5 & 221.76 & 31 & 0.17 & 0.17 & $>6.5 \times 10^{6}$ & - & \\
\hline $\begin{array}{l}\text { Farm G } \\
\text { (Pond 1) }\end{array}$ & 0.61 & 85 & 8.6 & 4 & & 31 & 0.05 & 0.05 & $>6.5 \times 10^{6}$ & Negative & $\begin{array}{l}\text { High } \\
\text { mortality rate }\end{array}$ \\
\hline
\end{tabular}

"Hardness from farm D, E, $\mathrm{F}$ and $\mathrm{G}$ were above $450 \mathrm{mg} \mathrm{l}^{-1} \mathrm{CaCo}_{3}$; All the ponds in farm $\mathrm{A}, \mathrm{B}, \mathrm{C}$ as well as ponds 1,2 and 3 in farm $\mathrm{D}$ were subjected to harvest due to WSSV outbreak in nearby ponds. Mortality rate of shrimps in farm D and F could not be observed due to emergency harvest

Table 4. Water quality parameters recorded in shrimp ponds in Elavur

\begin{tabular}{|c|c|c|c|c|c|c|c|c|c|c|c|c|c|}
\hline $\begin{array}{l}\text { Farm/ } \\
\text { Pond }\end{array}$ & $\begin{array}{l}\text { Pond } \\
\text { size } \\
\text { (ha) }\end{array}$ & $\begin{array}{l}\text { Stocking } \\
\text { density } \\
\left(\text { No. } \mathrm{m}^{-2}\right)\end{array}$ & $\begin{array}{l}\text { Sampling } \\
\text { No. }\end{array}$ & $\begin{array}{l}\text { Daysof } \\
\text { culture } \\
\text { (DOC) }\end{array}$ & $\mathrm{pH}$ & $\begin{array}{l}\text { Salinity } \\
(\%)\end{array}$ & $\begin{array}{l}\text { Alkalinity } \\
\mathrm{mg} \mathrm{l}^{-1}\end{array}$ & $\begin{array}{l}\text { Temperature } \\
\left({ }^{\circ} \mathrm{C}\right)\end{array}$ & $\begin{array}{l}\text { Ammonia } \\
\left(\mathrm{Mg} \mathrm{l}^{-1}\right)\end{array}$ & $\begin{array}{l}\text { Nitrite } \\
\left(\mathrm{mg} \mathrm{l}^{-1}\right)\end{array}$ & $\begin{array}{l}\text { Dissolved } \\
\text { oxygen } \\
\left(\mathrm{mg} \mathrm{l}^{-1}\right)\end{array}$ & $\begin{array}{l}\text { Vibrio load } \\
\left(\mathrm{CFU} \mathrm{m}{ }^{-1}\right)\end{array}$ & Remarks \\
\hline $\begin{array}{l}\text { Farm A } \\
\text { (Pond 1) }\end{array}$ & 0.40 & 99 & 1 & 17 & 7.9 & 20 & 200.16 & 31.0 & 0.20 & 0.01 & 07 & $5.4 \times 10^{5}$ & \\
\hline $\begin{array}{l}\text { Farm A } \\
\text { (Pond 2) }\end{array}$ & 0.28 & 99 & 1 & 17 & 8.1 & 20 & 210.17 & 31.0 & 0.20 & 0.01 & 07 & $4.2 \times 10^{5}$ & \\
\hline $\begin{array}{l}\text { Farm B } \\
\text { (Pond 1) }\end{array}$ & 0.40 & 82 & 1 & 10 & 7.6 & 10 & 175.14 & 31.0 & 0.20 & 0.01 & 12 & $6.0 \times 10^{4}$ & \\
\hline $\begin{array}{l}\text { Farm C } \\
\text { (Pond 1) }\end{array}$ & 0.40 & 50 & 1 & 27 & 8.3 & 20 & 275.22 & 31.0 & 0.20 & 0.01 & 05 & $2.2 \times 10^{5}$ & \\
\hline $\begin{array}{l}\text { Farm D } \\
\text { (Pond 1) }\end{array}$ & 0.40 & 74 & 1 & 30 & 8.0 & 13 & 250.20 & 31.0 & 0.20 & 0.01 & 07 & $1.8 \times 10^{5}$ & \\
\hline $\begin{array}{l}\text { Farm E } \\
\text { (Pond 1) }\end{array}$ & 0.20 & 84 & 1 & 27 & 8.1 & 21 & 350.28 & 31.0 & 0.20 & 0.01 & 05 & $7.8 \times 10^{5}$ & \\
\hline $\begin{array}{l}\text { Farm F } \\
\text { (Pond 1) }\end{array}$ & 0.40 & 61 & 1 & 22 & 8.3 & 19 & 290.23 & 31.0 & 0.20 & 0.01 & 05 & $4.8 \times 10^{5}$ & \\
\hline $\begin{array}{l}\text { Farm A } \\
\text { (Pond 1) }\end{array}$ & 0.40 & 99 & 2 & 34 & 7.6 & 16 & 200.16 & 30.0 & 0.20 & 0.01 & 09 & $2.4 \times 10^{6}$ & \\
\hline $\begin{array}{l}\text { Farm A } \\
\text { (Pond 2) }\end{array}$ & 0.28 & 99 & 2 & 34 & 7.9 & 18 & 250.20 & 30.0 & 0.20 & 0.01 & 09 & $1.9 \times 10^{6}$ & \\
\hline $\begin{array}{l}\text { Farm B } \\
\text { (Pond 1) }\end{array}$ & 0.40 & 82 & 2 & 27 & 7.6 & 10 & 175.14 & 30.0 & 0.20 & 0.01 & 12 & $7.2 \times 10^{5}$ & \\
\hline $\begin{array}{l}\text { Farm C } \\
\text { (Pond 1) }\end{array}$ & 0.40 & 50 & 2 & 44 & 8.3 & 20 & 315.25 & 30.0 & 0.20 & 0.05 & 05 & $1.5 \times 10^{6}$ & \\
\hline $\begin{array}{l}\text { Farm D } \\
\text { (Pond 1) }\end{array}$ & 0.40 & 74 & 2 & 47 & 7.9 & 10 & 300.24 & 30.0 & 0.20 & 0.05 & 12 & $1.6 \times 10^{6}$ & \\
\hline $\begin{array}{l}\text { Farm E } \\
\text { (Pond 1) }\end{array}$ & 0.20 & 84 & 2 & 44 & 7.9 & 18 & 320.26 & 30.0 & 0.60 & 0.50 & 05 & $3.2 \times 10^{6}$ & \\
\hline
\end{tabular}




\begin{tabular}{|c|c|c|c|c|c|c|c|c|c|c|c|c|c|}
\hline $\begin{array}{l}\text { Farm/ } \\
\text { Pond }\end{array}$ & $\begin{array}{l}\text { Pond } \\
\text { size } \\
\text { (ha) }\end{array}$ & $\begin{array}{l}\text { Stocking } \\
\text { density } \\
\left(\text { No. }^{-2} \text { ) }\right.\end{array}$ & $\begin{array}{l}\text { Sampling } \\
\text { No. }\end{array}$ & $\begin{array}{l}\text { Daysof } \\
\text { culture } \\
\text { (DOC) }\end{array}$ & $\mathrm{pH}$ & $\begin{array}{l}\text { Salinity } \\
(\%)\end{array}$ & $\begin{array}{l}\text { Alkalinity } \\
\mathrm{mg} \mathrm{l}^{-1}\end{array}$ & $\begin{array}{l}\text { Temperature } \\
\left({ }^{\circ} \mathrm{C}\right)\end{array}$ & $\begin{array}{l}\text { Ammonia } \\
\left(\mathrm{Mg} \mathrm{l}^{-1}\right)\end{array}$ & $\begin{array}{l}\text { Nitrite } \\
\left(\mathrm{mg} \mathrm{l}^{-1}\right)\end{array}$ & $\begin{array}{l}\text { Dissolved } \\
\text { oxygen } \\
\left(\mathrm{mg} \mathrm{l}^{-1}\right)\end{array}$ & $\begin{array}{l}\text { Vibrio load } \\
\left(\mathrm{CFU} \mathrm{ml} \mathrm{l}^{-1}\right)\end{array}$ & Remarks \\
\hline $\begin{array}{l}\text { Farm F } \\
\text { (Pond 1) }\end{array}$ & 0.40 & 61 & 2 & 39 & 7.9 & 20 & 250.20 & 30.0 & 0.01 & 0.01 & 09 & $1.8 \times 10^{5}$ & \\
\hline $\begin{array}{l}\text { Farm A } \\
\text { (Pond 1) }\end{array}$ & 0.40 & 99 & 3 & 50 & 7.8 & 15 & 225.18 & 32.0 & 1.00 & 0.50 & 05 & $2.5 \times 10^{6}$ & \\
\hline $\begin{array}{l}\text { Farm A } \\
\text { (Pond 2) }\end{array}$ & 0.28 & 99 & 3 & 50 & 7.8 & 15 & 290.23 & 32.0 & 1.00 & 0.50 & 05 & $2.4 \times 10^{6}$ & \\
\hline $\begin{array}{l}\text { Farm B } \\
\text { (Pond 1) }\end{array}$ & 0.40 & 82 & 3 & 43 & 7.7 & 10 & 160.13 & 32.0 & 0.40 & 0.25 & 07 & $1.8 \times 10^{6}$ & \\
\hline $\begin{array}{l}\text { Farm C } \\
\text { (Pond 1) }\end{array}$ & 0.40 & 50 & 3 & 60 & 7.6 & 15 & 210.17 & 32.0 & 0.60 & 0.50 & 05 & $6.5 \times 10^{6}$ & \\
\hline $\begin{array}{l}\text { Farm D } \\
\text { (Pond 1) }\end{array}$ & 0.40 & 74 & 3 & 63 & 7.2 & 12 & 350.28 & 32.0 & 0.60 & 1.00 & 07 & $1.7 \times 10^{6}$ & \\
\hline $\begin{array}{l}\text { Farm E } \\
\text { (Pond 1) }\end{array}$ & 0.20 & 84 & 3 & 60 & 7.8 & 15 & 290.23 & 32.0 & 0.40 & 0.50 & 07 & $3.2 \times 10^{6}$ & \\
\hline $\begin{array}{l}\text { Farm F } \\
\text { (Pond 1) }\end{array}$ & 0.40 & 61 & 3 & 57 & 7.6 & 16 & 260.21 & 32.0 & 0.01 & 0.01 & 05 & $1.1 \times 10^{6}$ & \\
\hline $\begin{array}{l}\text { Farm A } \\
\text { (Pond 1) }\end{array}$ & 0.40 & 99 & 4 & 67 & 7.7 & 19 & 225.18 & 31.5 & 1.00 & 1.00 & 05 & $1.6 \times 10^{6}$ & \\
\hline $\begin{array}{l}\text { Farm A } \\
\text { (Pond 2) }\end{array}$ & 0.28 & 99 & 4 & 67 & 7.9 & 19 & 325.26 & 31.5 & 1.00 & 1.00 & 03 & $1.4 \times 10^{6}$ & \\
\hline $\begin{array}{l}\text { Farm B } \\
\text { (Pond 1) }\end{array}$ & 0.40 & 82 & 4 & 60 & 7.8 & 10 & 150.12 & 31.5 & 0.50 & 0.25 & 07 & $4.2 \times 10^{5}$ & \\
\hline $\begin{array}{l}\text { Farm C } \\
\text { (Pond 1) }\end{array}$ & 0.40 & 50 & 4 & 77 & 7.1 & 22 & 300.24 & 31.5 & 2.00 & 0.50 & 03 & $1.6 \times 10^{6}$ & \\
\hline $\begin{array}{l}\text { Farm D } \\
\text { (Pond 1) }\end{array}$ & 0.40 & 74 & 4 & 80 & - & - & - & - & - & - & - & - & Harvested \\
\hline $\begin{array}{l}\text { Farm E } \\
\text { (Pond 1) }\end{array}$ & 0.20 & 84 & 4 & 77 & 7.3 & 20 & 275.22 & 31.5 & 1.00 & 1.00 & 03 & $1.6 \times 10^{6}$ & \\
\hline $\begin{array}{l}\text { Farm F } \\
\text { (Pond 1) }\end{array}$ & 0.40 & 61 & 4 & 74 & 8.0 & 20 & 250.20 & 31.5 & 0.01 & 0.01 & 05 & $4.4 \times 10^{5}$ & \\
\hline $\begin{array}{l}\text { Farm A } \\
\text { (Pond 1) }\end{array}$ & 0.40 & 99 & 5 & 81 & 7.1 & 20 & 215.17 & 30.0 & 1.00 & 1.00 & 05 & $9.8 \times 10^{5}$ & \\
\hline $\begin{array}{l}\text { Farm A } \\
\text { (Pond 2) }\end{array}$ & 0.28 & 99 & 5 & 81 & 7.1 & 20 & 280.22 & 30.0 & 0.60 & 1.00 & 05 & $1.2 \times 10^{6}$ & \\
\hline $\begin{array}{l}\text { Farm B } \\
\text { (Pond 1) }\end{array}$ & 0.40 & 82 & 5 & 74 & - & - & - & - & - & - & - & - & Harvested \\
\hline $\begin{array}{l}\text { Farm C } \\
\text { (Pond 1) }\end{array}$ & 0.40 & 50 & 5 & 91 & - & - & - & - & - & - & - & - & Harvested \\
\hline $\begin{array}{l}\text { Farm E } \\
\text { (Pond 1) }\end{array}$ & 0.20 & 84 & 5 & 91 & 7.8 & 24 & 240.19 & 30.0 & 0.60 & 1.00 & 05 & $1.7 \times 10^{6}$ & \\
\hline $\begin{array}{l}\text { Farm F } \\
\text { (Pond 1) }\end{array}$ & 0.40 & 61 & 5 & 88 & 8.0 & 22 & 280.22 & 30.0 & 0.50 & 0.50 & 05 & $7.0 \times 10^{5}$ & \\
\hline $\begin{array}{l}\text { Farm A } \\
\text { (Pond 1) }\end{array}$ & 0.40 & 99 & 6 & 102 & - & - & - & - & - & - & - & - & Harvested \\
\hline $\begin{array}{l}\text { Farm A } \\
\text { (Pond 2) }\end{array}$ & 0.28 & 99 & 6 & 102 & - & - & - & - & - & - & - & - & Harvested \\
\hline $\begin{array}{l}\text { Farm E } \\
\text { (Pond 1) }\end{array}$ & 0.20 & 84 & 6 & 112 & - & - & - & - & - & - & - & - & Harvested \\
\hline $\begin{array}{l}\text { Farm F } \\
\text { (Pond 1) }\end{array}$ & 0.40 & 61 & 6 & 109 & - & - & - & - & - & - & - & - & Harvested \\
\hline
\end{tabular}

alternately; high fluctuation in the concentration of DO was observed due to the presence of heavy algal blooms and lack of water quality management practices (Ajin et al.,
2016). Water quality management practices are universally recommended to maintain healthier culture (Wyk and Scarpa, 1999). 
A study done in $P$. vannamei, outlined high susceptibility to $V$. alginolyticus infection consequent to an increase in water temperature from 27 to $34^{\circ} \mathrm{C}$ (Cheng et al., 2005). This parameter has been reported by many researchers and has been found to be inter-related with other physicochemical characteristics (Cheng et al., 2005; Moser et al., 2012). In shrimp, low water temperature will directly affect metabolic rate and thereby decrease the rate of ammonia accumulation (Kir et al., 2004). Wyban et al. (1995) reported that the optimal temperature for $P$. vannamei growth is $27^{\circ} \mathrm{C}$. In the present study, the average temperature was $30^{\circ} \mathrm{C}$, which was well within the range of 26 to $33^{\circ} \mathrm{C}$ reported by Wickins and Lee (2008) for $P$. vannamei culture. Ferreira et al. (2011) reported that $24^{\circ} \mathrm{C}$ is the optimum temperature for $P$. vannamei farming in Brazil.

Although, shrimps are tolerant to euryhaline conditions, the optimal salinity for best growth, range between 15 to $25 \%$ o (Baliao, 2000). Low salinity often leads to undesirable changes in the environment (Wahab et al., 2003). Low salinities were observed in few ponds in Kalpakkam, which might be a factor contributing to shrimp stress. Increased mortality rate in shrimps were observed in farms $\mathrm{E}$ and $\mathrm{G}$ of Kalpakkam, which had lower salinity values than other ponds (Table 2 and 3). Shrimp ponds D4 and D5 which had the onset of WSSV outbreak were also found to have low salinity levels. Variations in salinity is a major factor affecting shrimp immune response and combined with variations in temperature and $\mathrm{pH}$, can influence WSSV outbreaks (Tendencia et al., 2011). Ramos-Carreno et al. (2014) reported that P. vannamei was less susceptible to WSSV at intermediate salinities of 15 - 28\%o. Our findings of low salinity affecting shrimp ponds, triggering WSSV outbreak, agree with these reports.

Another major factor for successful shrimp farming is optimum $\mathrm{pH}$. Pond water with $\mathrm{pH}$ in the range 6.5-9.0 is considered optimal for aquaculture production (Carbajal-Hernandez et al., 2012). High pH has been identified commonly in many shrimp ponds and is caused due to the removal of carbon dioxide for phytoplankton growth (Penmetsa et al., 2013). The estimated $\mathrm{pH}$ value in our study ranged between 7.1 to 8.9 and was within the permissible range reported for shrimp farming.

Hypoxia increases the susceptibility of cultured shrimps to imbibe WSSV infection (Lehmann et al., 2016). The tolerance to hypoxia was reported to vary between species but the effects start to appear when oxygen drops below $2 \mathrm{mgl}^{-1}$ (Diaz, 2001). The concentration of DO has been directly associated with the presence of algal bloom in ponds. Hypoxia reduces metabolic rate which results in mortality in fish (Shang et al., 2006) and shrimp (Wu et al.,
2002). The immune resistance can also be impacted under hypoxic conditions, which can lead to disease outbreak (Direkbusarakom and Danayadol, 1998). The current study revealed that all ponds had DO concentrations greater than $2 \mathrm{mg} \mathrm{l}^{-1}$. A previous study conducted in central India showed a variation of 3.0 to $8.3 \mathrm{mg} \mathrm{l}^{-1} \mathrm{DO}$ level in shrimp ponds (Mishra et al., 2008). In the present study, DO was found to be within the permissible range.

Alkalinity, another important parameter signifies the total sodium bicarbonates and carbonates present in the pond water. Apart from other parameters, alkalinity and hardness are relatively stable but can alter with time (Wurts and Durborow, 1992). A recent study suggested that increased alkalinity levels protect crustaceans from trace metal toxicity and the optimal level for alkalinity is $100 \mathrm{mg} \mathrm{l}^{-1}$ (Boyd et al., 2016). Calcium deposition on exoskeleton followed by slow growth in shrimps is reported when the $\mathrm{pH}$ is above 8.3 and alkalinity above $150 \mathrm{mg} \mathrm{l}^{-1}$ (Chanratchakool, 2003). Pond alkalinity tends to correlate with $\mathrm{pH}$, hardness and carbon dioxide concentration. Data from our shrimp ponds in India recorded alkalinity in the range of 100 to $350 \mathrm{mg} \mathrm{l}^{-1}$. In this study, it was observed that, WSSV positive ponds as well as non-infected ponds had alkalinity value greater than $150 \mathrm{mg} \mathrm{l}^{-1}$. The desired total alkalinity level for most of aquaculture species are reported to be within $50-150 \mathrm{mg} \mathrm{l}^{-1}$ but not less than $20 \mathrm{mg} \mathrm{l}^{-1}$ (Wurts, 2002). Based on these reports, the alkalinity levels in our study recorded an increase by $200 \mathrm{mg} \mathrm{l}^{-1}$, however these variations did not affect successful shrimp harvest.

Water hardness is an important factor in determining the pond water quality (Boyd et al., 2016). The proportion of divalent ions, as calcium and magnesium are major contributors to water hardness (Wurts and Durborow, 1992). Whilst Wyk et al. (1999) have reported optimal level to be greater than $150 \mathrm{mg} \mathrm{l}^{-1}$, while Ferreira et al. (2011) reported $1000 \mathrm{mg} \mathrm{l}^{-1} \mathrm{CaCO}_{3}$ equivalents. From our study, hardness quality was found to be uniformly high in all the shrimp ponds.

Optimal amount of ammonia and nitrite content in shrimp ponds are necessary to harvest a healthy crop. Accumulation of ammonia leads to toxicity and causes adverse side effects including slow growth, increased oxygen consumption and shrimp mortality (Chen and Kou, 1992). Increased levels of ammonia in water have been shown to affect the immunity of $P$. vannamei leading to susceptibility to $V$. alginolyticus infection (Liu and Chen, 2004). Other studies from India estimated ammonia level to be in the range of 0.02 to $2 \mathrm{mg} \mathrm{l}^{-1}$ among the southern region (Chakravarty et al., 2016) and 0.001 to $0.01 \mathrm{mg} \mathrm{l}^{-1}$ among the northern regions (Mudassir et al., 2016). 
Nitrite toxicity in $P$. vannamei has been extensively studied in terms of $\mathrm{LD}_{50}$, growth and survival (Lin and Chen, 2003), immunity, gut health and anti-oxidant capacity (Guo et al., 2013; Duan et al., 2018), as well as in disease resistance and related molecular mechanisms (Tseng and Chen, 2004; Guo et al., 2016) and are considered to cause chronic toxicity in shrimps. In our study of winter crop, initially nitrite concentrations were uniformly low in all ponds; however as the days of culture increased to 50 days, the nitrite concentration increased. This shows that accumulation of nitrite was continuous throughout the culture period; however, successful crops were harvested. Enhanced nitrite accumulation can lead to elevated levels of reactive oxygen species (ROS), DNA damage, cell apoptosis, oxidative stress and reductions in total haemocyte counts (THC) (Xian et al., 2011). Continuous imbalance in nitrite concentration is known to inhibit anti-oxidant enzymes needed for shrimp metabolism and growth (Roques et al., 2015). Tseng and Chen (2004) have also highlighted that water nitrite level caused lower immunity in P. vannamei triggering $V$. alginolyticus infection. Periodical removal of sludge from pond bottom has been recommended to diminish the level of total ammonia nitrogen (TAN) in water (Ajin et al., 2016). In general, it is recommended that the concentration of pond nitrite should remain below $2 \mathrm{mg} \mathrm{l}^{-1}$ (Romano and Zeng, 2013). The data from farms in Kalpakkam and Elavur revealed that nitrite concentration reaching to $1 \mathrm{mg} \mathrm{l}^{-1}$ levels did not considerably affect the ponds where successful shrimp harvest was carried out.

Vibriosis causes mass mortalities in both hatcheries and grow-out systems leading to high economic loss (Ananda Raja et al., 2017a, b). Many shrimp diseases like tail necrosis, loose shell syndrome (LSS), red disease, cuticular wounds, septic hepatopancreatitis and white gut disease (WGD) occur due to infections caused by Vibrio spp. (Ray et al., 2017). The infestations of these bacterial species are wide-spread and to maintain healthy stock, periodical assessments of vibrio load in pond water are necessary. Ferreira et al. (2011) reported Vibrio load level of $10 \mathrm{cfu} \mathrm{ml}^{-1}$ whereas the Vibrio load from the farms in the study area was relatively high. However, in our study, a few ponds with high Vibrio content tested negative for WSSV and did not result in WSSV outbreak.

WSSV has been known as a serious threat for shrimp cultivation (Thitamadee et al., 2016). Low water quality causes stress and lowers the shrimp immune system and can be the cause for outbreaks of lethal viruses like WSSV (Kautsky et al., 2000). Increase in viral load have been associated with pond water physicochemical characteristics including DO, ammonia, temperature and bacterial count (Zhang et al., 2016). In P. monodon, WSSV outbreaks were triggered by fluctuation in temperature coupled with Vibrio spp. load and low salinity (Tendencia et al., 2011; Ananda Raja et al., 2017a, b). Increased evidence of WSSV outbreak with extreme salinity conditions has been reported as well (Liu et al., 2006; Ramos-Carreno et al., 2014).

On screening the shrimps, four out of twenty ponds $(20 \%)$ in Kalpakkam area were positive for WSSV while ponds located in south Elavur region were not affected until harvest (Table 1, 2, 3 and 4). During this study, it was observed that ponds that were located adjacent to the infected ponds were also immediately harvested in an attempt to pre-empt the further spread of disease. For example, pond B1 in Kalpakkam tested negative for WSSV; however, an emergency harvest was carried out following a disease outbreak in nearby farms. In analysing the Elavur shrimp farming ponds, few parameters like ammonia, nitrite and Vibrio load were found to be higher than the optimal value, yet successful crop was harvested

In conclusion, optimal water physicochemical characteristics are important for shrimp health and for sustainable shrimp production, routine monitoring of shrimp ponds as well as adoption of pond management practices are essential.

\section{Acknowledgements}

We acknowledge the financial support of the Newton Fund Global Research Partnership in Aquaculture for the project 'Poverty alleviation through prevention and future control of the two major socioeconomically-important diseases in Asian aquaculture', by the Department of Biotechnology, Ministry of Science and Technology, India, under Sanction Order BT/IN/Indo-UK/BBSRCAqua/38/MSS/2015-16 and from the UK BBSRC, UK ESRC and UK Aid under contract BB/N005058/1.

\section{References}

Ajin, A. M., Silvester, R., Alexander, D., Nashad, M. and Abdulla, M. H. 2016. Characterisation of blooming algae and bloom-associated changes in the water quality parameters of traditional pokkali cum prawn fields along the south-west coast of India. Environ. Monit. Assess., 188: $1-10$.

Ananda Raja, R., Panigrahi, A. and Kumar, S. 2012a. Epidemiological investigation of brackishwater culture systems in West Bengal, India. J. Appl. Aquac., 24(1): 49-59. https://doi.org/10.1080/10454438.2012.652029.

Ananda Raja, R., Sujeet, K., Sundaray, J. K., Debasis, D., Biswas, G. and Ghoshal, T. K. 2012b. Hematological parameters in relation to sex, morphometric characters and incidence of white spot syndrome virus in tiger shrimp Penaeus monodon Fabricius, 1798 from Sunderban, West Bengal. Indian J. Fish., 59(4): 169-174. 
Ananda Raja, R., Panigrahi, A., De, D. and Kumar, S. 2017a. Investigations on white spot disease outbreak in Penaeus monodon (Fabricius, 1798) in association with Vibrio mimicus infection in the Sunderbans, West Bengal, India. Indian J. Fish., 64: 56-60.

Ananda Raja, R., Sridhar, R., Balachandran, C., Palanisammi, A., Ramesh, S. and Nagarajan, K. 2017b. Pathogenicity profile of Vibrio parahaemolyticus in farmed Pacific white shrimp, Penaeus vannamei. Fish Shellfish Immunol., 67: 368-381.

Bagarinao, T. and Flores, E. E. C. 1995. Towards sustainable aquaculture in South-east Asia and Japan, Proceedings of the Seminar-Workshop on Aquaculture Development in South-east Asia. 26-28 April 1994, Aquaculture Department. South-east Asian Fisheries Development Centre (SEAFDEC), Iloilo, Philippines.

Baliao, D. D. 2000. Environment-friendly schemes in intensive shrimp farming. Aquaculture Department, South-east Asian Fisheries Development Centre, Iloilo, Philippines, p. 19.

Biswas, G., Ananda Raja, R., De, D., Sundaray, J. K., Ghoshal, T. K., Shyne Anand, P. S., Sujeet Kumar, Panigrahi, A., Thirunavukkarasu, A. R. and Ponniah, A. G. 2012. Evaluation of production and economic returns of two brackishwater polyculture systems in tide-fed ponds. J. Appl. Ichthyol., 28: 116-122.

Boyd, C. E., Tucker, C. S. and Somridhivej, B. 2016. Alkalinity and hardness: Critical but elusive concepts in aquaculture. J. World Aquac. Soc., 47: 6-41. https://doi.org/10.1111/ jwas.12241.

Carbajal-Hernandez, J. J., Sanchez-Fernandez, L. P., Carrasco-Ochoa, J. A. and Martinez-Trinidad, J. F. 2012. Immediate water quality assessment in shrimp culture using fuzzy inference systems. Expert. Syst. Appl., 39: 10571-10582.

Chakravarty, M. S., Ganesh, P. R. C., Amarnath, D., Sudha, B. S. and Babu, T. S. 2016. Spatial variation of water quality parameters of shrimp (Litopenaeus vannamei) culture ponds at Narsapurapupeta, Kajuluru and Kaikavolu villages of East Godavari District, Andhra Pradesh. Int. J. Fish. Aquat. Stud., 4: 390-395.

Chanratchakool, P. 2003. Problems in shrimp culture during the wet season. Aquac. Asia., 8: 38-40.

Chen, J. and Kou, Y. 1992. Effects of ammonia on growth of Penaeus japonicus juveniles. Aquaculture, 104: 249-260. https://doi. org/10.1016/0044-8486(92)90207-2.

Cheng, W., Wang, L. U. and Chen, J. C. 2005. Effect of water temperature on the immune response of white shrimp Litopenaeus vannamei to Vibrio alginolyticus. Aquaculture, 250: 592-601. doi:10.1016/j.aquaculture. 2005.04.060.

Cohen, J. M., Samocha, T. M., Fox, J. M., Gandy, R. L. and Lawrence, A. L. 2005. Characterisation of water quality factors during intensive raceway production of juvenile Litopenaeus vannamei using limited discharge and biosecure management tools. Aquac. Eng., 32: 425-442. DOI: 10.1016/j.aquaeng.2004.09.005.

Diaz, R. J. 2001. Overview of hypoxia around the world. J. Environ. Qual., 30: 275-281. doi: 10.2134/jeq2001.302275x.

Direkbusarakom, S. and Danayadol, Y. 1998. Effect of oxygen depletion on some parameters of the immune system in black tiger shrimp (Penaeus monodon). Advances in shrimp Biotechnology, National Centre for Genetic Engineering and Biotechnology, Bangkok, Thailand, p. 147-149.

Duan, Y., Liu, Q., Wang, Y., Zhang, J. and Xiong, D. 2018. Impairment of the intestine barrier function in Litopenaeus vannamei exposed to ammonia and nitrite stress. Fish Shellfish Immunol., 78: 279-288. doi: 10.1016 /j.fsi.2018.04.050.

Durand, S., Lightner, D. V., Nunan, L. M., Redman, R. M., Mari, J. and Bonami, J. R. 1996. Application of gene probes as diagnostic tools for white spot baculovirus (WSBV) of penaeid shrimp. Dis. Aquat. Org., 27: 59-66.

Ferreira, N. C., Bonetti, C. and Seiffert, W. Q. 2011 Hydrological and water quality indices as management tools in marine shrimp culture. Aquaculture, 318(3-4): 425-433. https://doi.org/10.1016/j.aquaculture.2011.05.045.

Flegel, T. W. 2012. Historic emergence, impact and current status of shrimp pathogens in Asia. J. Invertebr. Pathol., 11: 166-173. doi: 10.1016/j.jip.2012.03.004.

Gopal, S., Otta, S. K., Kumar, S., Karunasagar, I., Nishibuchi, M. and Karunasagar, I. 2005. The occurrence of Vibrio species in tropical shrimp culture environments; Implications for food safety. Int. J. Food Microbiol., 102: 151-159. doi: 10.1016/j.ijfoodmicro.2004.12.011.

Guo, H., Xian, J. A., Li, B., Ye, C. X., Wang, A. L., Miao, Y. T. and Liao, S. A. 2013. Gene expression of apoptosis-related genes, stress protein and antioxidant enzymes in hemocytes of white shrimp Litopenaeus vannamei under nitrite stress. Comp. Biochem. Physiol. Part C, Toxicol. Pharmacol., 157: 366-371. DOI: 10.1016/j.cbpc.2013.03.001.

Guo, H., Xian, J. A. and Wang, A. L. 2016. Analysis of digital gene expression profiling in hemocytes of white shrimp Litopenaeus vannamei under nitrite stress. Fish Shellfish Immunol., 56: 1-11. DOI: 10.1016/j.fsi.2016.06.059.

Hopkins, J. S., Hamilton, R. D., Sandier, P. A., Browdy, C. L. and Stokes, A. D. 1993. Effect of water exchange rate on production, water quality, effluent characteristics and nitrogen budgets of intensive shrimp ponds. J. World. Aquac. Soc., 24: 304-320.

Kautsky, N., Ronnback, P., Tedengren, M. and Troell, M. 2000. Ecosystem perspectives on management of disease in shrimp pond farming. Aquaculture, 191(1-3): 145-161.

Kir, M., Kumlu, M. and Eroldogan, O. T. 2004. Effects of temperature on acute toxicity of ammonia to Penaeus semisulcatus juveniles. Aquaculture, 241(1-4): 479-489. DOI: 10.1016/j.aquaculture.2004.05.003. 
Kumaran, M., Anand, P. R., Kumar, J. A., Ravisankar, T., Paul, J., Vasagam, K. P. K., Vimala, D. D. and Raja, K. A. 2017. Is Pacific white shrimp (Penaeus vannamei) farming in India is technically efficient? - A comprehensive study. Aquaculture, 468: 262-270.

Le Moullac, G. and Haffner, P. 2000. Environmental factors affecting immune responses in crustacea. Aquaculture, 191(1-3): 121-131. https://doi.org/10.1016/ S0044-8486(00)00422-1.

Lehmann, M., Schleder, D. D., Guertler, C., Perazzolo, L. M. and Vinatea, L. 2016. Hypoxia increases susceptibility of Pacific white shrimp to whitespot syndrome virus (WSSV). Braz. Arch. Vet. Med. Anim. Sci., 68(2): 397-403 (In Portuguese).

Lin, Y. C. and Chen, J. C. 2003. Acute toxicity of nitrite on Litopenaeus vannamei (Boone) juveniles at different salinity levels. Aquaculture, 224: 193-201. https://doi. org/10.1016/S0044-8486(03)00220-5.

Liu, B., Yu, Z., Song, X., Guan, Y., Jian, X. and He, J. 2006. The effect of acute salinity change on white spot syndrome (WSS) outbreaks in Fenneropenaeus chinensis, Aquaculture, 253(1-4): 163-170. DOI: 10.1016/j.aquaculture.2005.08.022.

Liu, C. H. and Chen, J. C. 2004. Effect of ammonia on the immune response of white shrimp Litopenaeus vannamei and its susceptibility to Vibrio alginolyticus. Fish Shellfish Immunol., 16(3): 321-334. doi: 10.1016/S1050-4648(03) 00113-X.

Mishra, R. R., Rath, B. and Thatoi, H. 2008. Water quality assessment of aquaculture ponds located in Bhitarkanika mangrove ecosystem, Orissa, India. Turk. J. Fish. Aquat. Sci., 77(1): 71-77.

MPEDA 2016. MPEDA Annual report 2015-2016. Marine Products Export Development Authority, Kochi, Kerala, India. https://mpeda.gov.in/MPEDA/annual_reports.php\#.

Moser, J. R., Alvarez, D. A. G., Cano, F. M., Garcia, T. E., Molina, D. E. C., Clark, G. P., Marques, M. R. F., Barajas, F. J. M. and Lopez, J. H. 2012. Water temperature influences viral load and detection of white spot syndrome virus (WSSV) in Litopenaeus vannamei and wild crustaceans. Aquaculture, 326: 9-14.

Mudassir, A., Mohite, A. S. and Rahul, S. 2016. Soil and water quality parameters of brackishwater shrimp farms of Ratnagiri, Maharashtra. Asian J. Anim. Sci., 11: 107-110. DOI: 10.15740/HAS/TAJAS/11.2/107-110.

Penmetsa, A. R. K. R., Muppidi, S. R., Popuri, R., Golla, S. B. and Tenneti, R. 2013. Impact of aquaculture on physico-chemical characteristics of water and soils in the coastal tracts of East and West Godavari Districts, Andhra Pradesh, India. Int. J. Eng. Trends Technol., 6: 313-319.

Ramos-Carreno, S., Valencia-Yanez, R., Correa-Sandoval, F., Ruiz-Garcia, N., Diaz-Herrera, F. and Giffard-Mena, I. 2014. White spot syndrome virus (WSSV) infection in shrimp (Litopenaeus vannamei) exposed to low and high salinity. Arch. Virol., 159(9): 2213-2222. doi: 10.1007/ s00705-014-2052-0.

Ray, A. K., Gopal, C., Solanki, H. G., Ravisankar, T. and Patil, P. K. 2017. Effect of orally administered vibrio bacterin on immunity, survival and growth in tiger shrimp (Penaeus monodon) grow-out culture ponds. J. Appl. Microbiol., 65(6): 475-481. https://doi.org/10.1111/ lam.12802.

Romano, N. and Zeng, C. 2013. Toxic effects of ammonia, nitrite and nitrate to decapod crustaceans: A review on factors influencing their toxicity, physiological consequences and coping mechanisms. Rev. Fish. Sci. Aquac., 21(1): 121. DOI: $10.1080 / 10641262.2012 .753404$.

Roques, J. A. C., Schram, E., Spanings, T., Van Schaik, T., Abbink, W., Boerrigter, J. and De Vries, P. 2015. The impact of elevated water nitrite concentration on physiology, growth and feed intake of African catfish Clarias gariepinus (Burchell 1822). Aquac. Res., 46(6): 1384-1395.

Sanchez-Martinez, J. G., Aguirre-Guzman, G. and Mejia-Ruiz, H. 2007. White spot syndrome virus in cultured shrimp: A review. Aquac. Res., 38(13): 1339-1354. https://doi. org/10.1111/j.1365-2109.2007.01827.x.

Shang, E. H. H., Yu, R. M. K. and Wu, R. S. S. 2006. Hypoxia affects sex differentiation and development, leading to a male-dominated population in zebrafish (Danio rerio). Environ. Sci. Technol., 40(9): 3118-3122. doi: 10.1021/ es0522579.

Tendencia, E. A., Bosma, R. H. and Verreth, J. A. J. 2010. WSSV risk factors related to water physico-chemical properties and microflora in semi-intensive Penaeus monodon culture ponds in the Philippines. Aquaculture, 302(3-4): 164-168. DOI: 10.1016/j.aquaculture.2010.03.008.

Tendencia, E. A., Bosma, R. H. and Verreth, J. A. 2011. White spot syndrome virus (WSSV) risk factors associated with shrimp farming practices in polyculture and monoculture farms in the Philippines, Aquaculture, 311(1-4): 87-93.

Thitamadee, S., Prachumwat, A., Srisala, J., Jaroenlak, P., Salachan, P. V., Sritunyalucksana, K., Flegel, T. W. and Itsathiphaisarn, O. 2016. Review of current disease threats for cultivated penaeid shrimp in Asia. Aquaculture, 452: 69-87.

Tseng, I. T. and Chen, J. C. 2004. The immune response of white shrimp Litopenaeus vannamei and its susceptibility to Vibrio alginolyticus under nitrite stress. Fish Shellfish Immunol., 17(4): 325-333. doi: 10.1016/j.fsi.2004.04.010.

Wahab, M. A., Bergheim, A. and Braaten, B. 2003. Water quality and partial mass budget in extensive shrimp ponds in Bangladesh. Aquaculture, 218: 413-423. DOI: 10.1016/ s0044-8486(03)00009-7.

Wickins, J. F. and Lee, D. O. C. 2008. Crustacean farming: Ranching and culture, $2^{\text {nd }}$ edn. Wiley-Blackwell, Iowa, USA, 464 pp. 
Wu, R. S. S., Lam, P. K. S. and Wan, K. L. 2002. Tolerance to and avoidance of hypoxia by the penaeid shrimp Metapenaeus ensis. Environ. Pollut., 118(3): 351-355. doi: 10.1016/ s0269-7491(01)00298-6.

Wurts, W. A. and Durborow, R. M. 1992. Interactions of pH, carbon dioxide, alkalinity and hardness in fish ponds. SRAC Publication No. 464. Southern Regional Aquaculture Centre, Iowa State University, Iowa, USA, p. 1-4.

Wurts, W. A. 2002. Alkalinity and hardness in production ponds. World Aquac., 33(1): 16-17.

Wyban, J., Walsh, W. A. and Godin, D. M. 1995. Temperature effects on growth, feeding rate and feed conversion of the Pacific white shrimp Penaeus vannamei. Aquaculture, 138(1-4): 267-279. https://doi.org/10.1016/0044-8486(95) 00032-1.

Wyk, P. V., Davis-Hodgkins, M., Laramore, R., Main, K. L., Moutain, J. and Scarpa, J. 1999. Farming marine shrimp in recirculating freshwater systems. Harbor Branch
Oceanographic Institute, Florida Atlantic University, Florida, USA, 229 pp.

Wyk, P. V. and Scarpa, J. 1999. Water quality requirements and management. In: Wyk, P. V., Davis-Hodgkins, M., Laramore, R., Main, K. L., Moutain, J. and Scarpa, J. (Eds.), Farming marine shrimp in recirculating freshwater systems, Harbor Branch Oceanographic Institute, Florida Atlantic University, Florida, USA, p. 141-162.

Xian, J. A., Wang, A. L., Chen, X. D., Gou, N. N., Miao, Y. T., Liao, S. A. and Ye, C. X. 2011. Cytotoxicity of nitrite on haemocytes of the tiger shrimp, Penaeus monodon, using flow cytometric analysis. Aquaculture, 317(1-4): 240-244.

Zhang, J. S., Li, Z. J., Wen, G. L., Wang, Y. L., Luo, L., Zhang, H. J. and Dong, H. B. 2016. Relationship between white spot syndrome virus (WSSV) loads and characterisations of water quality in Litopenaeus vannamei culture ponds during the tropical storm. Iran. J. Vet. Res., 17(3): $210-214$ 